\title{
Physiological and transcriptomic analyses to reveal underlying phenolic acid action in consecutive monoculture problem of Polygonatum odoratum
}

\author{
Xianzhi $\mathrm{Ni}^{1}$, Chenzhong Jin ${ }^{1}$, Aiyu Liu ${ }^{2}$, Yong Chen ${ }^{{ }^{*}}$ and Yihong $\mathrm{Hu}^{1 *}$
}

\begin{abstract}
Background: The root rot of fragrant solomonseal (Polygonatum odoratum) has occurred frequently in the traditional $P$. odoratum cultivating areas in recent years, causing a heavy loss in yield and quality. The phenolic acids in soil, which are the exudates from the P. odoratum root, act as allelochemicals that contribute to the consecutive monoculture problem (CMP) of the medicinal plant. The aim of this study was to get a better understanding of $P$. odoratum CMP.

Results: The phenolic acid contents, the nutrient chemical contents, and the enzyme activities related to the soil nutrient metabolism in the first cropping (FC) soil and continuous cropping (CC) soil were determined, and the differentially expressed genes (DEGs) related to the regulation of the phenolic acids in roots were analyzed. The results showed that five low-molecule-weight phenolic acids were detected both in the CC soil and FC soil, but the phenolic acid contents in the CC soil were significantly higher than those in the FC soil except vanillic acid. The contents of the available nitrogen, available phosphorus, and available potassium in the CC soil were significantly decreased, and the activities of urease and sucrase in the CC soil were significantly decreased. The genomic analysis showed that the phenolic acid anabolism in P. odoratum in the CC soil was promoted. These results indicated that the phenolic acids were accumulated in the CC soil, the nutrient condition in the CC soil deteriorated, and the nitrogen metabolism and sugar catabolism of the CC soil were lowered. Meantime, the anabolism of phenolic acids was increased in the CC plant.
\end{abstract}

Conclusions: The CC system promoted the phenolic acid anabolism in P. odoratum and made phenolic acids accumulate in the soil.

Keywords: Fragrant solomonseal, Root rot, Continuous cropping soil, First cropping soil, Low-molecule-weight phenolic acids, Genomic analysis

\footnotetext{
*Correspondence: henon@163.com; huyhongwangyi@163.com

1 Collaborative Innovation Center for Field Weeds Control of Hunan Province,

Hunan University of Humanities, Science and Technology, Loudi 417000,

China

Full list of author information is available at the end of the article
}

C C The Author(s). 2021 Open Access This article is licensed under a Creative Commons Attribution 4.0 International License, which permits use, sharing, adaptation, distribution and reproduction in any medium or format, as long as you give appropriate credit to the original author(s) and the source, provide a link to the Creative Commons licence, and indicate if changes were made. The images or other third party material in this article are included in the article's Creative Commons licence, unless indicated otherwise in a credit line to the material. If material is not included in the article's Creative Commons licence and your intended use is not permitted by statutory regulation or exceeds the permitted use, you will need to obtain permission directly from the copyright holder. To view a copy of this licence, visit http://creativecommons.org/licenses/by/4.0/. The Creative Commons Public Domain Dedication waiver (http://creativecommons.org/publicdomain/zero/1.0/) applies to the data made available in this article, unless otherwise stated in a credit line to the data. 


\section{Background}

Polygonatum odoratum (Mill.) Druce, popularly known as fragrant solomonseal, is a traditional Chinese perennial medicinal plant mainly cultivated in the southern parts of China and the other Southeast Asian countries such as Thailand and Vietnam. As a medicinal and edible plant, its rhizome has the functions of removing dryness, promoting secretion, and quenching thirst [1, 2], and it is widely welcomed especially in the Southeast Asian markets. However, the root rot of $P$. odoratum has occurred frequently in the planting areas in recent years due to the long-term continuous cropping (CC), causing the serious consecutive monoculture problem (CMP) with a sharp decline both in yield and quality. For example, in Shaodong County of Hunan Province, which was the largest traditional high-quality $P$. odoratum planting area in China, the medicinal peasants have almost given up the cultivation of $P$. odoratum due to this serious replant disease.

Researches have revealed that CMP is mainly caused by three factors covering the imbalance of soil nutrients, the shift in microorganisms towards pathogenic and allelopathic autotoxicity, and the root exudates of secondary metabolites, among which the root exudates are likely to be considered as the main factor to induce the root rot by influencing rhizosphere microbes and soil nutrients [3]. The root exudates include low-moleculeweight substances such as polysaccharides, vitamins, nucleotides, and phenolic acids. Among them, phenolic acids are most likely to be considered as the allelochemicals that can change membrane permeability, inhibit nutrient uptake, and inactivate plant endogenous hormones to influence the normal physiological process [4].

Phenolic acids are a kind of substance containing active organic acids on their aromatic rings that are mainly synthesized by the shikimic acid pathway which provides phenylpropanoids or synthesized by the polyketide pathway in plants which provides simple phenolic acids directly. Shikimic acid is synthesized from 4-phosphate erythritose via the phosphoenolpyruvic acid and pentose phosphate pathway, and then the aromatic amino acids are subsequently used as precursors for the synthesis of phenolic acids [5]. Phenolic acids in plants are products responding to environmental stresses, and they may be involved in the intracellular and intercellular signaling processes as signal transporting substances [6]. When plant roots are infected, the phenolic acid levels in the root cells are promoted to act as antioxidants and inhibit infectious microorganisms directly [7].

Although phenolic acids are of vital importance for the plant's interaction with the environment, few documents have been published on the relationship between the $P$. odoratum phenolic acids and the root rot disease. An early study showed that the low-molecule-weight substances extracted from the $P$. odoratum rhizosphere soil solutions exhibited an obvious self-poison effect on $P$. odoratum seedling growth and allelopathy effect on the rhizome spore germination rate [8]. Our recent studies have revealed that the Polygonatum CMP could be alleviated or eradicated under the rotation regimes or with an aeroponic system $[9,10]$, and the miRNAs-regulated genes participating in the sugar and phenylpropanoid metabolism have been enriched through the mircoRNA sequencing on the $\mathrm{CC}$ and the first cropping (FC) P. odoratum [11]. All these results prompted that the root rot in $P$. odoratum was likely related to the phenolic acid metabolism. Other researchers revealed that the root exudates including phenolic acids may not only involve in the signal transduction and hormone synthesis but also modify the rhizosphere soil environment to regulate the microbial community in the rhizosphere in some other medicinal plants such as Rehmannia glutinosa [5, 12]. Besides, recent researches have provided more information on the complex correlation among the root rot, phenolic acids, and CMP. Although phenolic acids in the CC soils stimulate the propagation of Fusarium which directly results in root rot, single phenolic acid also plays a role as a double-edged sword to decrease the Fusarium growth and production at a lower level, and the CC system can strengthen the phenolic acid accumulation in the rhizosphere soils of peanut, Panax notoginseng, and Chrysanthemum morifolium [13-15].

In this study, the low-molecule-weight phenolic acids from the rhizosphere soil of FC and CC P. odoratum were identified, and the relative chemical and physiological indices for evaluating the influences of the phenolic acids on the soil were also determined. Furthermore, the transcriptome profiles from the FC and $\mathrm{CC}$ rhizomes of $P$. odoratum were compared through high-throughput sequencing, and then the differentially expressed genes (DEGs) were analyzed. Then the pathway related to the phenolic acid metabolism was analyzed using the Kyoto Encyclopedia of Genes and Genomes (KEGG). This research might provide more information to understand the regulatory function of the phenolic acids in $P$. odoratum under the CC system.

\section{Results}

\section{Rhizosphere soil phenolic acids}

The rhizosphere soil phenolic acids were determined using the high-performance liquid chromatography (HPLC) method as compared to the standard samples of $p$-hydroxybenzoic acid, vanillic acid, syringic acid, cumaric acid, and ferulic acid with the retention time of $15.42,19.29,21.84,27.16$, and $28.94 \mathrm{~min}$, respectively (Fig. S1A), and the above five phenolic acids were detected both in the FC and CC rhizosphere soil (Fig. S1B, Fig. S1C). $R^{2}$ of the five regression curves was 0.999 (Table 1). The good linearity indicated that the determination method was reliable. The phenolic acid 
Table 1 Regression equations of phenolic acid standard samples using external standard method

\begin{tabular}{llll}
\hline Phenolic acid standard sample & Regression equation & $\mathbf{R}^{\mathbf{2}}$ & Retention time (min) \\
\hline -hydroxybenzoic acid & $y=19.394 x+0.5019$ & 0.999 & 15.42 \\
Vanillic acid & $y=19.040 x+0.0441$ & 0.999 & 19.29 \\
Syringic acid & $y=29.678 x-0.0005$ & 0.999 & 21.84 \\
Cumaric acid & $y=42.687 x+0.3221$ & 0.999 & 27.16 \\
Ferulic acid & $y=27.663 x-0.0114$ & 0.999 & 28.94 \\
\hline
\end{tabular}

contents in the FC and CC rhizosphere soil were calculated according to the equations in Table 1 . As shown in Table 2, $p$-hydroxybenzoic, syringic acid, cumaric acid, and ferulic acid contents in the $\mathrm{CC}$ soil were significantly higher than those in the FC soil, whereas vanillic acid in the CC and FC soil had no significant difference, although vanillic acid contents in the CC and FC soil exhibited much higher level than those of the other phenolic acids. The results showed that the phenolic acid contents in the CC soil were higher than those in the FC soil.

\section{Rhizosphere soil chemical properties and enzyme activities}

The rhizosphere soil elements of the CC and FC system reflecting the soil nutritional status were determined in this experiment. As shown in Table 3, the contents of total phosphorus (TP), total potassium (TK), available nitrogen (AN), available phosphorus (AP), available potassium (AK) in the CC soil were significantly lower than those in the FC soil, whereas the total nitrogen (TN) contents between the CC soil and FC soil had no significant change. Although TN in the CC soil showed no significant difference as compared to that in the FC soil, $\mathrm{AN}$, which is the form of nitrogen that can be absorbed directly by plants, decreased significantly, and the other nutrient chemicals also decreased significantly. These results insisted that the $\mathrm{CC}$ soil nutritional status had deteriorated.

The rhizosphere soil enzymes related to the soil nutrient metabolism level were also determined in this experiment. As shown in Table 4, the activities of polyphenol oxidase (PPO), catalase (CAT), and acid phosphatase (ACP) had no significant differences between the CC soil and FC soil, whereas the activities of urease (UE) and sucrase (SC) in the CC soil were significantly lower than those in the FC soil. The results indicated that the soil oxidative metabolism and phosphorus utilization had not been changed between the CC soil and FC soil, but the levels of the nitrogen metabolism and sugar catabolism of the CC soil were lowered as compared to the FC soil.

\section{RNA-seq analysis and qRT-PCR verification}

After filtering out the low-quality sequences, 299,730,812 clean reads, which were accounted for 95.38\% of the raw reads, were obtained from the samples of the CC and FC P. odoratum roots, and Q20 and Q30 values and $\mathrm{GC}$ contents were all in the reliable ranges (Table S1). Owing to the absence of the reference genome, the total clean reads were spliced to obtain 842,213 transcripts and 510,970 unigenes, and the N50 lengths of the transcripts and unigenes were 931 and $1167 \mathrm{bp}$, respectively, and 298,939 unigenes were annotated in the databases including NR, NT, KO, SwissProt, PFAM, GO, and KOG, whereas 176,156 unigenes were annotated in the GO database. Then a total number of 15,788 DEGs were screened out in the CC vs FC root tissues including 4843 up-regulated DEGs and 10,945 down-regulated DEGs (padj < 0.05).

These DEGs were significantly enriched in 307 GO functional items. The biological processes included "oxidation-reduction process", "carbohydrate metabolic process", "polysaccharide metabolic process", etc.; the molecular functions included "catalytic activity", "oxidoreductase activity", "hydrolase activity", etc.; the cell components included "cell wall", "cell periphery", "cytoskeletal part", etc. (Fig. 1A). The up-regulated DEGs were mainly enriched in the metabolic process, the single-organism metabolic process, the catalytic activity, the oxidoreductase activity, etc. (Fig. 1B-D); the downregulated DEGs were mainly enriched in the oxidationreduction process, the carbohydrate metabolic process, the hydrolase activity (acting on glycosyl bonds), the

Table 2 Phenolic acid contents in FC and CC rhizosphere soil

\begin{tabular}{llllll}
\hline Rhizosphere soil sample & $\boldsymbol{p}$-hydroxybenzoic acid $(\boldsymbol{\mu g} / \mathbf{g})$ & $\begin{array}{l}\text { Vanillic acid } \\
(\boldsymbol{\mu g} / \mathbf{g})\end{array}$ & $\begin{array}{l}\text { Syringic acid } \\
(\boldsymbol{\mu g} / \mathbf{g})\end{array}$ & $\begin{array}{l}\text { Cumaric acid } \\
(\boldsymbol{\mu g} / \mathbf{g})\end{array}$ & $\begin{array}{l}\text { Ferulic acid } \\
(\boldsymbol{\mu g} / \mathbf{g})\end{array}$ \\
\hline FC & 0.0554 & 0.2806 & 0.0337 & 0.0204 & 0.0187 \\
CC & $0.1372^{\mathrm{a}}$ & 0.3003 & $0.0590^{\mathrm{a}}$ & $0.0385^{\mathrm{a}}$ & $0.0303^{\mathrm{a}}$ \\
\hline
\end{tabular}

Note: ${ }^{a}$ was marked for the significant difference within the same column with the T-Student's method $(P<0.05, n=3)$. FC stands for first cropping, and CC stands for continuous cropping 
Table 3 Chemical properties in FC and CC rhizosphere soil

\begin{tabular}{lllllll}
\hline Rhizosphere soil sample & TN $(\mathbf{g} / \mathbf{k g})$ & TP $(\mathbf{g} / \mathbf{k g})$ & TK $(\mathbf{g} / \mathbf{k g})$ & AN $(\mathbf{m g} / \mathbf{k g})$ & AP $(\mathbf{g} / \mathbf{k g})$ & AK $(\mathbf{m g} / \mathbf{k g})$ \\
\hline FC & $1.953 \pm 0.027$ & $0.355 \pm 0.015^{\mathrm{a}}$ & $1.362 \pm 0.009^{\mathrm{a}}$ & $83.650 \pm 3.031^{\mathrm{a}}$ & $0.259 \pm 0.027^{\mathrm{a}}$ & $34.740 \pm 1.119^{\mathrm{a}}$ \\
CC & $2.038 \pm 0.045$ & $0.293 \pm 0.015$ & $1.177 \pm 0.228$ & $69.650 \pm 3.031$ & $0.226 \pm 0.052$ & $25.087 \pm 1.132$
\end{tabular}

Note: values were expressed as mean \pm standard error, and ${ }^{\text {a }}$ was marked for the significant difference within the same column with the T-Student's method $(P<$ $0.05, n=3)$. FC stands for first cropping, and CC stands for continuous cropping. TN stands for total nitrogen, TP stands for total phosphorus, TK stands for total potassium, AN stands for available nitrogen, AP stands for available phosphorus, and AK stands for available potassium

hydrolase activity, the external encapsulating structure, the cell periphery, etc. (Fig. 1E-G). The results showed that the gene expressions of the $\mathrm{CC}$ plant were lower than those of the FC plant on the whole level, but the responses of $P$. odoratum to CMP were regulated by complicated networks, and lots of DEGs took part in the processes such as the secondary metabolism and the response reaction. It should be mentioned that even the up-regulated DEGs and the down-regulated DEGs coexisted in the same processes. For example, although 665 DEGs were significantly down-regulated in the carbohydrate metabolic process, the number of significantly up-regulated DEGs was also up to 177 (Table S2).

The KEGG enrichment analysis showed that DEGs were mainly enriched in the metabolic and biosynthetic pathways including phenylpropanoid biosynthesis, linoleic acid metabolism, phenylalanine metabolism, and starch and sucrose metabolism pathway, etc. The upregulated DEGs were significantly enriched in the pathways such as "phenylalanine, tyrosine and tryptophan biosynthesis", "phenylalanine metabolism", "phenylpropanoid biosynthesis", and "nitrogen metabolism" (Fig. 2A), whereas the down-regulated DEGs were significantly enriched in the pathways such as "plant hormone signal transduction", "DNA replication", "brassinosteroid biosynthesis", and "sesquiterpenoid and triterpenoid biosynthesis" (Fig. 2B).

To eliminate those DEGs with relatively lower expressions, $\log _{2}$ foldchange $>1.5$ was selected as the screening condition, the pathways closely relative to the phenolic acid metabolism were enriched including "pentose phosphate pathway", "pentose and glucoronate interconversions", "starch and sucrose metabolism", "tyrosine metabolism, and phenylalanine", "tyrosine, and tryptophan biosynthesis" (Fig. S2), and then the significantly enriched DEGs were found including FBP, PGD, rpe, GPD, SORD, malZ, $g l g C$, E3.2.1.4, aroK, aroC, TYDC, and E1.10.3.1 (Table 5). These DEGs were classified into two groups: down-regulated DEGs and up-regulated DEGs. Among them, FBP, PGD, rpe, SORD, malZ, aroK, aro $C$, and TYDC were up-regulated, whereas $g l g C$, GPI, E3.2.1.4, and E1.10.3.1 were down-regulated (Fig. 3). Among these genes, it was found that the genes that encoded the enzymes in favor of the phenolic acid synthesis were up-regulated, whereas those unfavorable for the phenolic acid synthesis were down-regulated.

To verify the above results, a total of 12 up-regulated DEGs in the CC vs FC plants related to the synthesis of phenolic acids were selected from Table 5 and were tested at the mRNA expression level. The expression levels of DEGs after normalization were presented, and then their mRNA expressions of these DEGs were verified using the quantitative real-time PCR (qRT-PCR). As shown in Fig. 4, the mRNA levels of 11 DEGs were increased except that of $60,288.238790$. These results proved that the enriched pathways related to the phenolic acid anabolism through RNA-seq analysis in this study were reliable.

\section{Discussion}

The soil phenolic acids are usually released by plant leaching, root exudation, or decomposition of plant residues $[16,17]$. For the low-molecule-weight phenolic acids, there are two groups including benzoic acid derivatives and cinnamic acid derivatives, which play an important role in plant growth and interactions with the environment. These molecules reduce water utilization, hydraulic conductivity, and nutrient uptake to influence the plant's physiological state [18]. In this study, five low-molecule-weight phenolic acids were identified from the $P$. odoratum rhizosphere soil, i.e., $p$-hydroxybenzoic acid, vanillic acid, syringic acid, cumaric acid, and ferulic acid, where $p$-hydroxybenzoic acid, vanillic acid, and syringic acid were from benzoic acid derivatives, and cumaric acid and ferulic acid were from cinnamic acid derivatives. Although their total contents were very

Table 4 Soil enzyme activities in FC and CC rhizosphere soil

\begin{tabular}{llllll}
\hline Rhizosphere soil sample & PPO $(\mathbf{m g} / \mathbf{d} / \mathbf{g})$ & CAT $(\boldsymbol{\mu m o l} / \mathbf{d} / \mathbf{g})$ & UE $(\boldsymbol{\mu g} / \mathbf{d} / \mathbf{g})$ & ACP $(\boldsymbol{\mu m o l} / \mathbf{d} / \mathbf{g})$ & SC $(\mathbf{m g} / \mathbf{d} / \mathbf{g})$ \\
\hline FC & $49.269 \pm 0.438$ & $17.867 \pm 1.022$ & $392.069 \pm 32.173^{\mathrm{a}}$ & $22.871 \pm 0.830$ & $34.800 \pm 0.188^{\mathrm{a}}$ \\
CC & $47.727 \pm 0.688$ & $17.284 \pm 1.026$ & $298.174 \pm 14.843$ & $22.004 \pm 0.321$ & $20.801 \pm 0.177$ \\
\hline
\end{tabular}

Note: values were expressed as mean \pm standard error, and ${ }^{a}$ was marked for the significant difference within the same column with the T-Student's method $(P<$ $0.05, \mathrm{n}=3$ ). FC stands for first cropping, and CC stands for continuous cropping. PPO stands for polyphenol oxidase, CAT stands for catalase, UE stands for urease, ACP stands for acid phosphatase, and SC stands for sucrase 


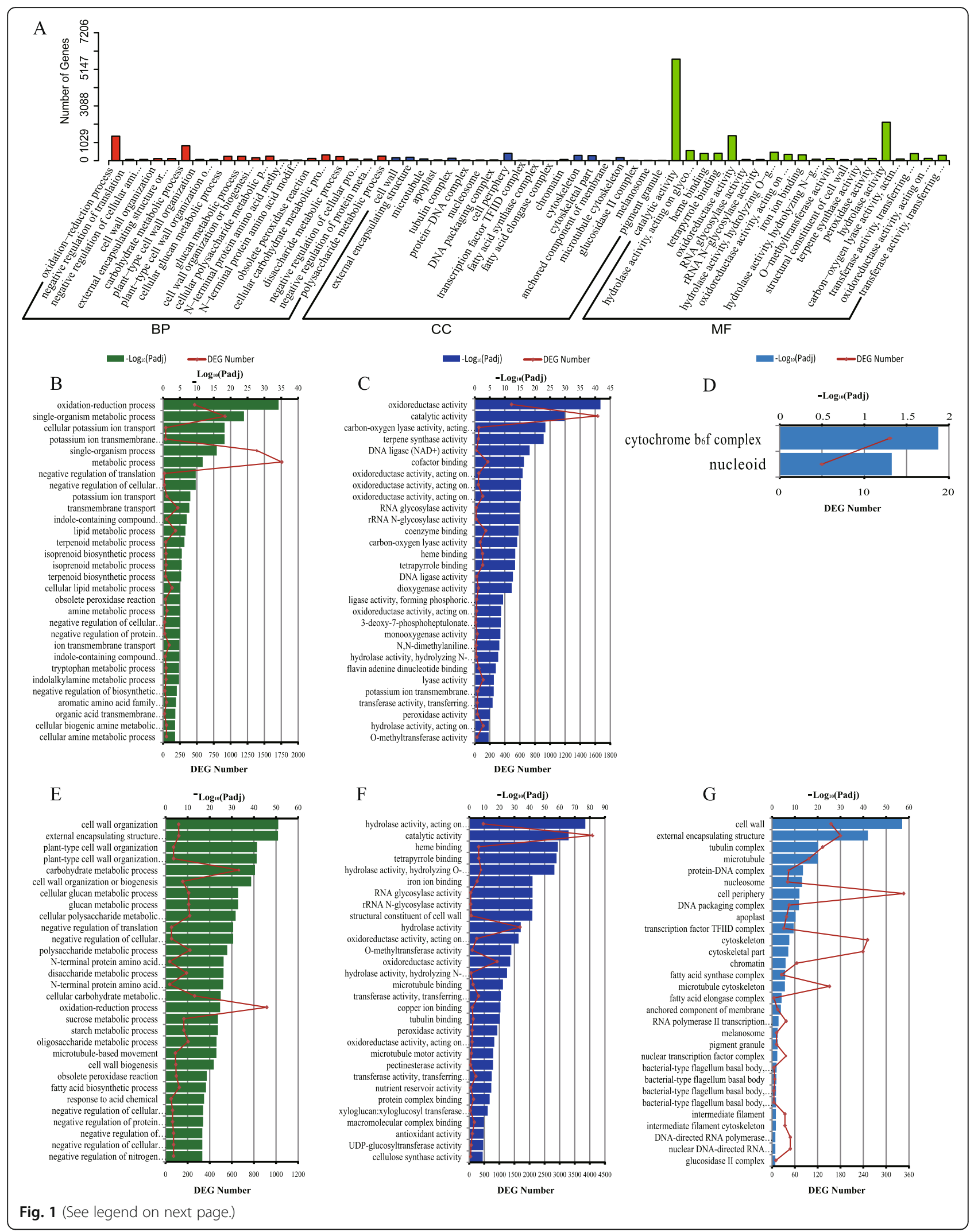


(See figure on previous page.)

Fig. 1 GO enrichments of DEGs in CC vs FC root tissues of Polygonatum odoratum. DEGs stands for differentially expressed genes. FC stands for first cropping, and CC stands for continuous cropping. A: DEGs in CC vs FC root tissues of Polygonatum odoratum, B - D: up-regulated DEGs in biological process, molecular function, and cellular component, respectively; E-G: down-regulated DEGs in biological process, molecular function, and cellular component, respectively. In Fig. 1A, BP stands for biological process, CC stands for cellular component, and MF stands for molecular function

subtle in the soil, the phenolic acid contents in the CC soil were significantly higher than those in the FC soil. These results supported that the CC system might result in an accumulation of phenolic acids secreted by the medicinal plant.

The content level and balance of nitrogen, phosphorus, and potassium in soil are essential for plant growth and development [19]. It was observed that TP, TK, AN, AP, and AK were significantly reduced in the CC soil. Subsequently, this phenomenon might lead to nutrient deterioration in the $\mathrm{CC}$ soil for medicinal plant growth. Soil AN is the nitrogen form which is easy to be absorbed and used directly by plants, mainly including $\mathrm{NH}_{4}^{+}{ }_{4} \mathrm{~N}, \mathrm{NO}^{-}{ }_{3}-\mathrm{N}$, amino acid, amide, and hydrolysable protein nitrogen [20]. The early research had proved that nitrogen in soil inhibits the formation of phenolic acids in plants, and there was a negative correlation between the total phenolic acids and the soil nitrogen content according to the "carbon/nutrient balance hypothesis" [21]. It was also observed this correlation in our experiment. The result showed that the AN in the CC soil was significantly lower than that in the FC soil, whereas the phenolic acids in the $\mathrm{CC}$ soil were at a higher level.

The phenolic acids are detrimental to the rhizosphere soil environment and can change the soil microbial community [9]. Phenolic acids have a hydroxyl group and a carboxyl group. For this reason, they can hydrogen-bond with the soil enzymes and change the soil $\mathrm{pH}$ value to worsen the soil's physiological status. The activities of soil enzymes can reflect the soil health status [22]. Soil SC hydrolyzes sucrose into monosaccharides, which are closely related to soil nutrition, whereas soil UE hydrolyzes urea to produce ammonia, which is the source of AN. In this experiment, it was found that the activities of UE and SC in the CC soil were decreased as compared to those in the FC soil. Under this situation, the utilization of sugar and the production of AN were weakened in the CC soil, and then the lack of nitrogen could lead to a comparatively higher content of phenolic acids in the CC plant as compared to that in the FC plant. Consequently, more phenolic acids were secreted and finally accumulated in the $\mathrm{CC}$ soil.

The high-throughput sequencing and relative qRTPCR analysis also revealed that the gene expressions of the enzymes that catalyzed the key steps in the synthesis of the phenolic acids were regulated in the P. odoratum root tissues. The pentose pathway and relative pathways are the major source for metabolic intermediates in plants [23] and can provide erythrose-4-phosphate as a precursor for shikimic acid. In the study, 12 kinds of DEGs that were closely relative to the shikimic acid and

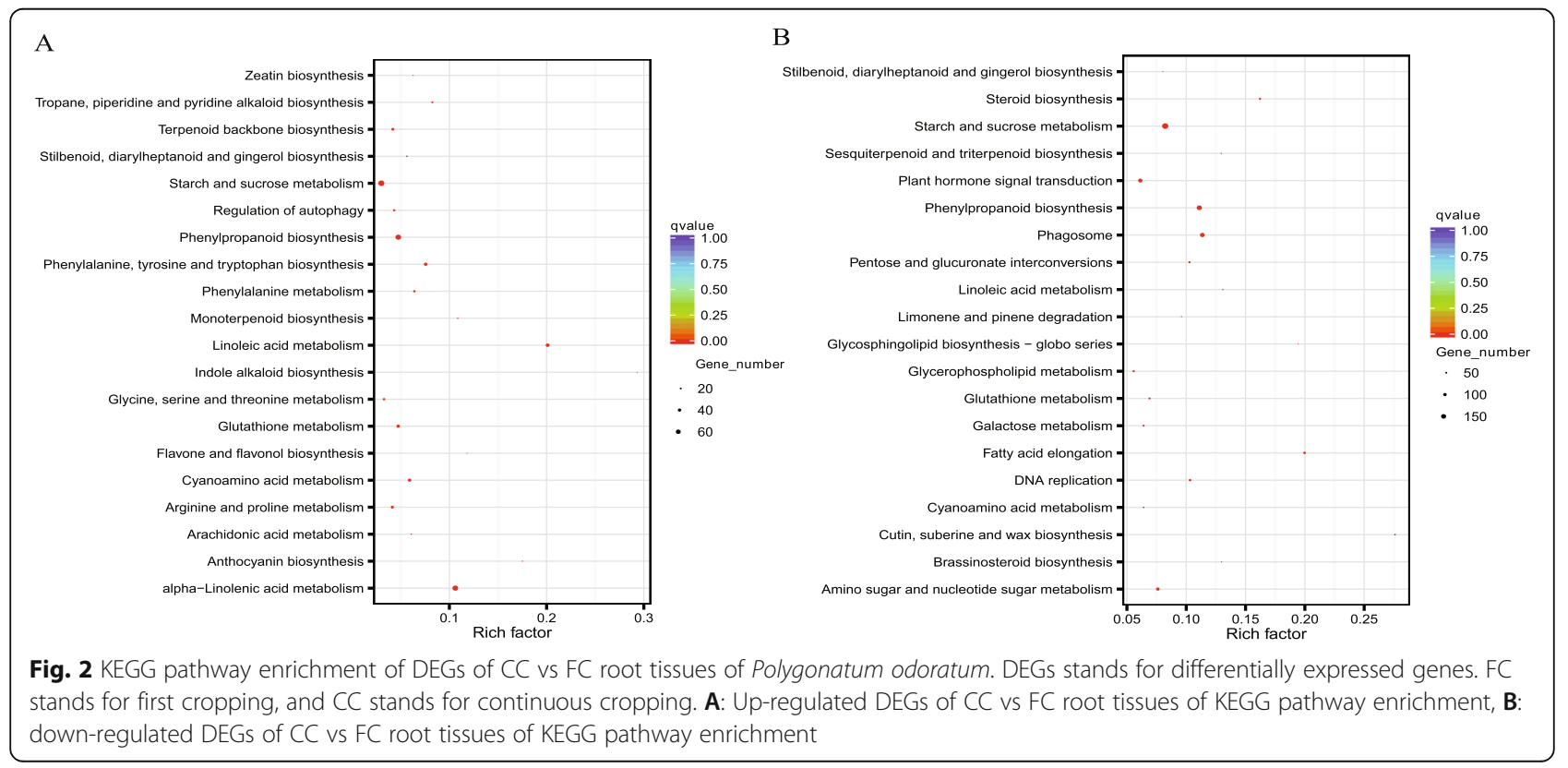


Table 5 Enriched DEGs relative to phenolic acid metabolism in CC vs FC root tissues of Polygonatum odoratum

\begin{tabular}{|c|c|c|c|c|}
\hline Pathway & Gene annotation & DEGs & | $\log _{2}$ foldchange| & Regulation \\
\hline \multirow[t]{5}{*}{ Pentose phosphate pathway } & FBP: fructose-1,6-biphospatase [EC:3.1.3.11] & $60,288.238790$ & 1.8774 & + \\
\hline & & $60,288.235250$ & 1.6639 & + \\
\hline & $\begin{array}{l}\text { PGD: 6-phosphogluconate dehydrogenase [EC: } \\
\text { 1.1.1.44] }\end{array}$ & $60,288.211711$ & 1.5588 & + \\
\hline & rpe: ribulose-phosphate 3-epimerase [EC:5.1.3.1] & $60,288.218462$ & 1.9319 & + \\
\hline & GPI: glucose-6-phosphate isomerase [EC:5.3.1.9] & $60,288.243910$ & 2.0490 & - \\
\hline \multirow[t]{3}{*}{ Pentose and glucoronate interconversions } & SORD: L-iditol 2-dehydrogenase [EC:1.1.1.14] & $60,288.223504$ & 1.8309 & + \\
\hline & & $60,288.223506$ & 1.6717 & + \\
\hline & & $60,288.218324$ & 1.7043 & + \\
\hline \multirow[t]{12}{*}{ Starch and sucrose metabolism } & malz: a-glucosidase [EC:3.2.1.20] & $60,288.201647$ & 3.9725 & + \\
\hline & $\begin{array}{l}\text { glgC: glucose-1-phosphate adenylyltransferase [EC: } \\
\text { 2.7.7.27] }\end{array}$ & $60,288.33753$ & 3.1600 & - \\
\hline & & $60,288.33754$ & 2.7498 & - \\
\hline & & $60,288.206885$ & 2.1132 & - \\
\hline & E3.2.1.4: endoglucanase [EC:3.2.1.4] & $60,288.14730$ & 4.4889 & - \\
\hline & & $60,288.10931$ & 5.5413 & - \\
\hline & & $60,288.10932$ & 6.4114 & - \\
\hline & & $60,288.10933$ & 6.1401 & - \\
\hline & & $60,288.10934$ & 7.8762 & - \\
\hline & & $60,288.148605$ & 1.8496 & - \\
\hline & & $60,288.148607$ & 2.5051 & - \\
\hline & & $60,288.148608$ & 1.9175 & - \\
\hline \multirow{8}{*}{$\begin{array}{l}\text { Phenylalanine, tyrosine and tryptophan } \\
\text { biosynthesis }\end{array}$} & aroK: shikimate kinase [EC:2.7.1.71] & $60,288.274987$ & 1.6112 & + \\
\hline & & $60,288.274989$ & 2.4732 & + \\
\hline & & $60,288.241945$ & 1.9399 & + \\
\hline & aroC: chorismate synthase [EC:4.2.3.5] & $60,288.232788$ & 1.6419 & + \\
\hline & & $60,288.230454$ & 1.5993 & + \\
\hline & & $60,288.239452$ & 1.8285 & + \\
\hline & & $60,288.239453$ & 1.7613 & + \\
\hline & & $60,288.239454$ & 1.8178 & + \\
\hline \multirow[t]{5}{*}{ Tyrosine metabolism } & TYDC: tyrosine decarboxylase [EC:4.1.1.25] & $60,288.348666$ & 3.8613 & + \\
\hline & & $60,288.348669$ & 4.0964 & + \\
\hline & & $60,288.198712$ & 3.1611 & + \\
\hline & E1.10.3.1: polyphenol oxidase [EC:1.10.3.1] & $60,288.244462$ & 2.3452 & - \\
\hline & & $60,288.367167$ & 2.5857 & - \\
\hline
\end{tabular}

Note: DEGs stands for differentially expressed genes. FC stands for first cropping, and CC stands for continuous cropping. + stands for up-regulation, and - stands for down-regulation

chorismate metabolisms were enriched, where eight of them were up-regulated, and four of them were downregulated (Fig. 5).

Among the up-regulated genes, $P D G$, rpe, and $S O R D$ encoded 6-phosphogluconate dehydrogenase, ribulose-phosphate 3-epimerase, and L-iditol 2dehydrogenase, respectively, and they were the enzymes for synthesis of ribulose-5-phosphate, xylulose- 5-phosphate, and xylusose, respectively [24-26]. The up-regulation of these genes was beneficial to the xylusoe-5-phosphate biosynthesis. FBP and malZ encoded fructose-1, 6-bisphosphatase and $\alpha$ glucosidase, respectively. The up-regulation of them was favorable for the synthesis of fructose-6phosphate, which was material for the synthesis of erthrose-4-phosphate, a precursor of shikmic acid [27, 


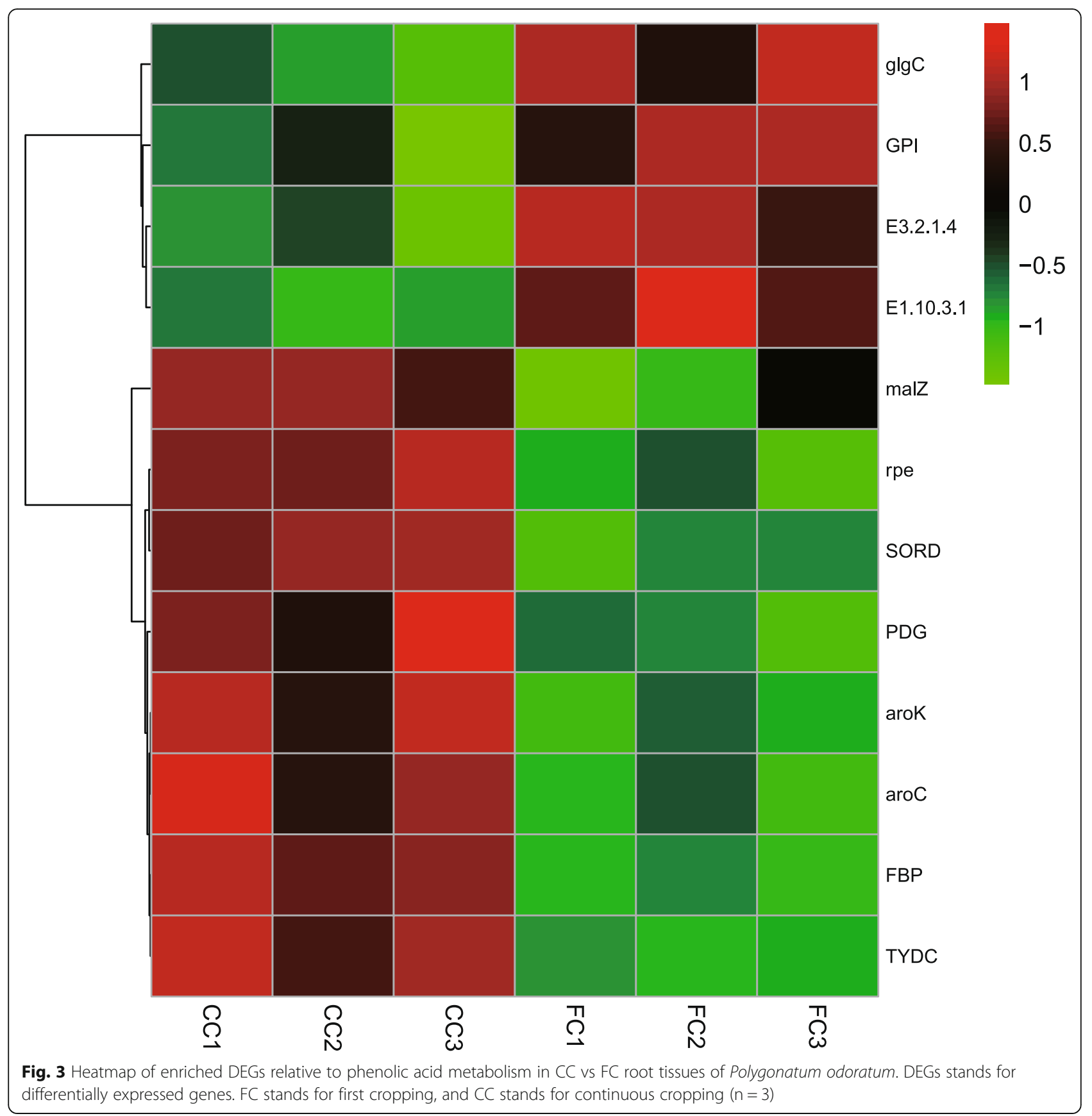

28]. aroK and $a r o C$ encoded shikimate kinase and chorismate synthase, respectively $[29,30]$, and the upregulation of them was beneficial to chorismate biosynthesis, which was the precursor of the lowmolecule-weight phenolic acids. On the contrary, the down-regulation of $g l g C, E 3.2 .1 .4$, and GPI, which encoded glucose-1-phosphate adenylyltransferase, endoglucanase, and glucose-6-phosphate isomerase, respectively, could inhibit the cellodexitrin and amylose metabolism [31-33]. This process in turn could provide more materials for the erthrose-4-phosphate biosynthesis. Early researches have proved that the phenolic acid biosynthesis in plants is triggered by biotic and abiotic stresses [34, 35]. Likewise, the results in our study also supported that the biosynthesis of phenolic acids in the $\mathrm{CC}$ plant proceeded through the activation of the primary pathway (shikimic acid) and the suppression of the relative branch pathway (cellodexitrin and amylose).

Tyramine is the product of tyrosine decarboxylation, and partial phenolic acids in plants can be conjugated with tyramine especially when the plant is under stress 


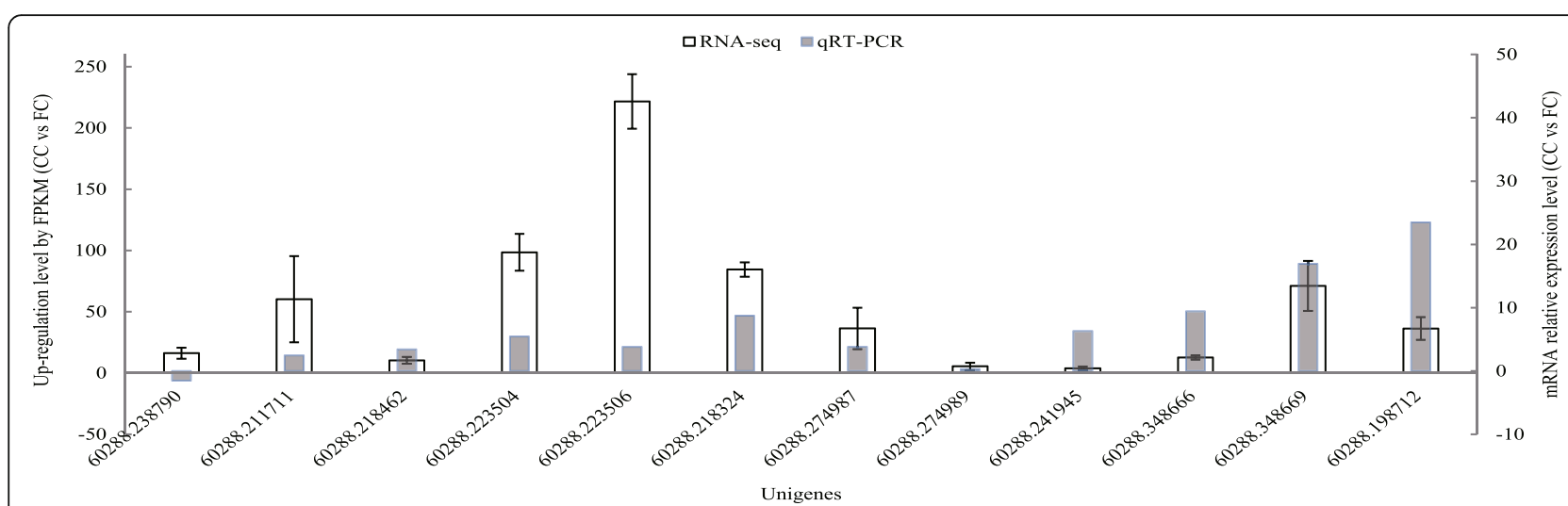

Fig. 4 Up-regulated DEGs expression level and their mRNA expression level of CC and FC root tissues. FC stands for first cropping, and CC stands for continuous cropping

[36, 37]. The tyramine-conjugated phenolic compounds act as phytoalexins to make plants adapt to the external stress, and the biosynthetic processes including the shikimate, phenylpropanoid, and arylmonoamine pathways are activated [38]. The conjugation is also favorable for more phenolic acids to accumulate. In this experiment, it was also found that the expression of tyrosine decarboxylase in the $\mathrm{CC}$ plant was up-regulated and polyphenol oxidase was down-regulated. It was speculated that the change might help to promote the phenolic acid anabolism in P. odoratum in the CC soil and made them accumulate in the soil.

The inducement of the root rot of $P$. odoratum in the $\mathrm{CC}$ soils was very complicated. The multiple factors to

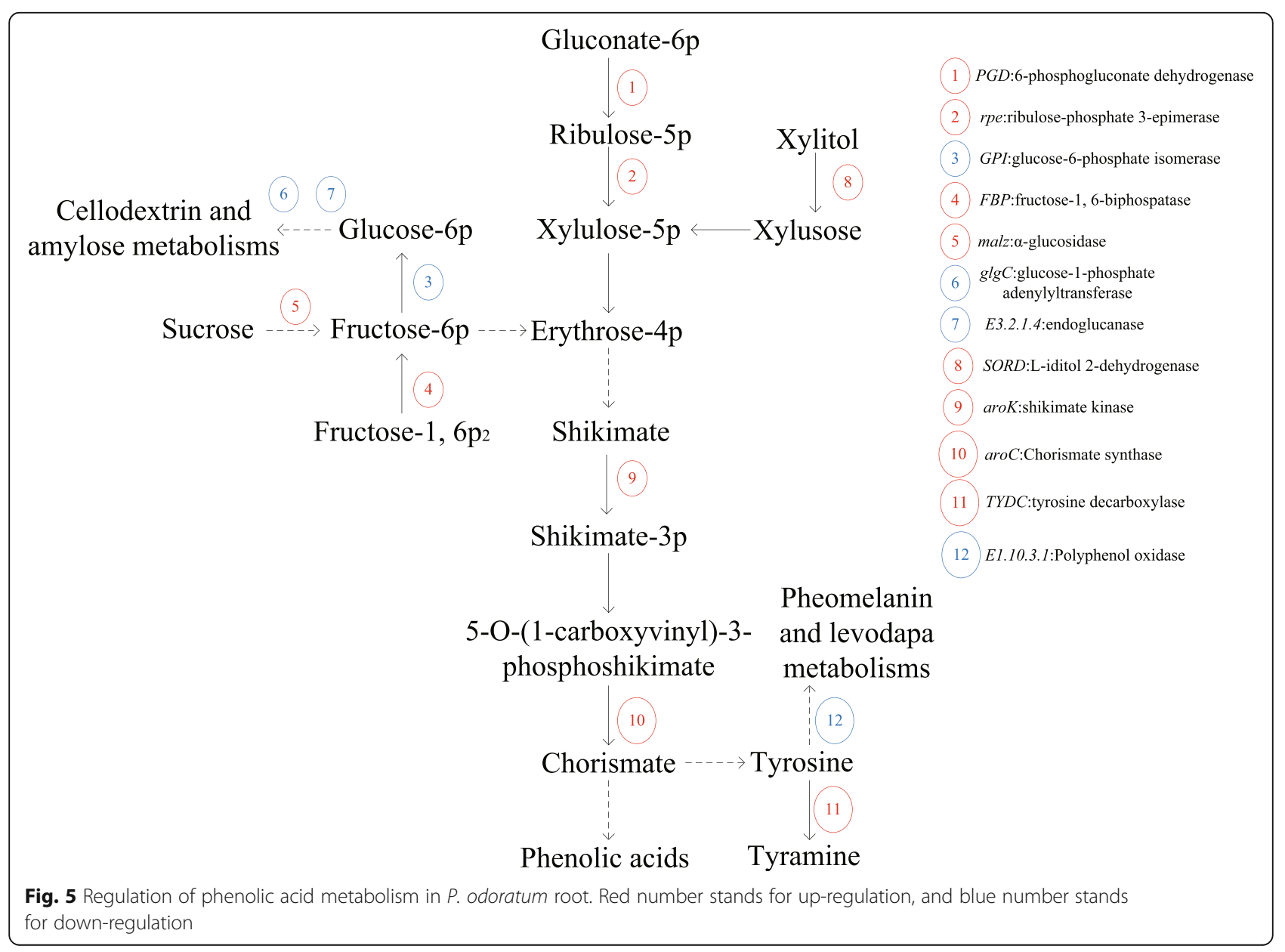


cause CMP may mainly include soil element imbalance, soil microbial population transforming to harmful population, and autotoxicity caused by the secretions from plant roots, but the comprehensive mechanism for CMP remains unclear [3]. For P. odoratum in the CC soils, the nutrient composition and the soil enzymes promoted the synthesis of phenolic acids. On the other hand, there might be multiple gene families activated together to accelerate the phenolic acid synthesis process coordinately to influence the CMP formation.

\section{Conclusions}

In this study, five low-molecule-weight phenolic acids were identified from the $P$. odoratum rhizosphere soil. Among them, the phenolic acid contents in the CC soil were significantly higher than those in the FC soil except vanillic acid. The contents of $\mathrm{AN}, \mathrm{AP}$, and $\mathrm{AK}$ in the $\mathrm{CC}$ soil were significantly higher than those in the FC soil, and the nitrogen metabolism and sugar catabolism of the CC soil were lowered. The genomic analysis showed that the phenolic acid anabolism in P. odoratum in the $\mathrm{CC}$ soil was promoted. This research presented us with a preliminary understanding of the role of phenolic acids in the CMP of P. odoratum. In perspective, proteomics and metabolomics are needed to look into more metabolic mechanism details of phenolic acid metabolism on the root rot of $P$. odoratum.

\section{Methods}

\section{Experiment materials and chemicals}

The P. odoratum cultivar "Zhushiwei" was selected as the experimental material and authenticated by associate Professor Zefa Liu, a horticulturist from Loudi Agricultural Institute, Hunan Province, China. The roots and rhizosphere soil of $P$. odoratum were collected in May, 2017 from the Gutang Town Experimental Station of Loudi City, Hunan Province, China with the permission of Loudi Agricultural Institute, Hunan Province, China. For the CC system, P. odoratum was cultivated in the land where the same plants had been harvested; for the FC system, $P$. odoratum was cultivated on the same date in the land near $\mathrm{CC}$ where the cabbages had been harvested. The collection of rhizosphere soil was according to Riley and Barber's method [39, 40]. Briefly, the soil with a complete root system was excavated in the selected plot, the large soil without root was shaken off gently, and then the soil adhered to the root circumference $(0-5 \mathrm{~mm}$ from the root circumference) was collected with a brush as the rhizosphere soil. The rhizosphere soil was air-dried under room temperature, passed through the $2 \mathrm{~mm}$ sieve, and stored at $4{ }^{\circ} \mathrm{C}$ until use.

Methanol, $p$-hydroxybenzoic acid, vanillic acid, syringic acid, cumaric acid, ferulic acid, and methanol were of chromatographic purity, and the other chemicals were of analytical purity.

\section{Rhizosphere soil phenolic acid determination}

The rhizosphere soil of $1.0 \mathrm{~g}$ was added with $3.0 \mathrm{~mL} 1 \mathrm{~N}$ $\mathrm{NaOH}$, and then shaken at $4{ }^{\circ} \mathrm{C}$ overnight. After centrifuged with $8000 \times \mathrm{g}$ at $4{ }^{\circ} \mathrm{C}$ for $10 \mathrm{~min}$, the supernatant was adjusted to $\mathrm{pH} 2.5$ with $\mathrm{HCl}$. After the liquid was extracted two times with $10 \mathrm{~mL}$ ethyl acetate, it was concentrated to dryness under nitrogen, and then dissolved in methanol. The solution was filtered with a $0.45 \mu \mathrm{m}$ membrane for the HPLC analysis.

The HPLC conditions were as follows: volume of $10 \mu \mathrm{L}$ was loaded on a Kromasil $\mathrm{C}_{18}$ column $(250 \mathrm{~mm} \times$ $4.6 \mathrm{~mm} \times 5 \mu \mathrm{m})$ (Akzo Nobel, Amsterdam, Netherlands) with a Rigol HPLC L3000 (Rigol Technologies, Beijing, China). The gradient elution was with eluent A $1 \%$ phosphoric acid and eluent $B$ methanol. The elution gradients were $80 \% \mathrm{~A}+20 \% \mathrm{~B}$ on $0-10 \mathrm{~min}, 70 \% \mathrm{~A}+30 \% \mathrm{~B}$ on $10-20 \mathrm{~min}, 50 \% \mathrm{~A}+50 \% \mathrm{~B}$ on $20-30 \mathrm{~min}, 50 \% \mathrm{~A}+$ $50 \% \mathrm{~B}$ on $30-40 \mathrm{~min}, 80 \% \mathrm{~A}+20 \% \mathrm{~B}$ on $40-45 \mathrm{~min}$, and $80 \% \mathrm{~A}+20 \% \mathrm{~B}$ on $45-55 \mathrm{~min}$, respectively, with the rate of $1 \mathrm{~mL} / \mathrm{min}$ at $30^{\circ} \mathrm{C}$, and the signal was detected at 280 $\mathrm{nm}$ with an ultraviolet detector. For the standard curve, $p$-hydroxybenzoic acid, vanillic acid, syringic acid, cumaric acid, and ferulic acid were used as standard samples and detected as above. The external standard method was used for the quantitative analysis of the phenolic acids as per Wang et al. [41].

\section{Determination of rhizosphere soil chemical properties and enzyme activity}

The TN content of the rhizosphere soil was determined with the Kjeldahl method, the TP content of the rhizosphere soil was determined with the molybdenum-blue colorimetry after the soil was extracted with the mixture of concentrated sulfuric acid and perchloric acid, the TK content of the rhizosphere soil was determined with the flame spectrophotometry after the soil was extracted with the mixture of concentrated sulfuric acid and perchloric acid, the AN content of the rhizosphere soil was determined with the diffusion method after hydrolysis with the alkaline solution, the AP content of the rhizosphere soil determined with the molybdenum-blue colorimetry after the soil was extracted with sodium hydroxide, and the AK content of the rhizosphere soil was determined with the flame spectrophotometry after the soil was extracted with sodium acetate [42]. The PPO activity of the rhizosphere soil was determined with the catechol method [43]. The CAT activity of the rhizosphere soil was determined as per Kraus and Fletcher [44]. The UE activity of the rhizosphere soil was determined with the indophenol blue colorimetric method [45]. The ACP of the rhizosphere soil activity was 
determined as per Saa et al. [46]. The SC activity of the rhizosphere soil was determined as per $\mathrm{Li}$ and $\mathrm{Lu}$ [47]. All of these analyses were assigned using the kits of Suzhou Comin Biotechnology Co. Ltd. and were repeated three times at the biological sample level.

\section{RNA-seq and bioinformatics analysis of root}

The root samples of CC and FC plants were obtained randomly from the fields during the rhizome expansion stage, and each tissue had three biological repeats in total. The library preparation and sequencing were performed in the Novogene Technology Co., Ltd. (Beijing, China). The total RNA was extracted using the Invitrogen Kit (Thermo Fisher Scientific, Beijing, China) and detected with a nanophotometer (Implen Inc., CA, USA). The sequencing libraries were generated using NEBNext Ultra RNA Library Prep Kit for Illumina (New England Biolabs, MA, USA). The libraries were sequenced on Illumina Hiseq Platform and then $150 \mathrm{bp}$ paired-end reads were generated.

The clean reads were obtained from the raw pair-end reads of mRNA sequencing data by removing adapters, low-quality reads, and the reads in which bases could not be determined, and then Trinity was employed to splice the clean-reads to obtain the de novo transcriptome assembly [48]. For DEGs analysis, DESeq R package(1.10.1)was used to screen the differential expression $(n=3)$ with the gene symbol annotation (padj <0.05) [49]. The KEGG pathway enrichment analysis was performed using KOBAS (2.0) and the pathway with false discovery rate $(F D R) \leq 0.05$ was defined as the significantly enriched pathway with DEGs [50].

\section{qRT-PCR analysis}

The total RNA from the P. odoratum root was extracted with Trizol reagent according to the manufacture's instruction. To obtain cDNA, the total RNA $(0.5 \mu \mathrm{g})$ was reversely transcribed using a TUREscript cDNA Synthesis Kit (Aidlab Biotechnologies Co., Ltd., Beijing, China) with oligo $\mathrm{dT}$ as a primer. The candidate genes and the reference gene were analyzed with a qTOWER2.2 realtime PCR (Analytik Jena AG, Jena, Thuringia, Germany) with the primers in Table S3. The protocol for qRT-PCR was as follows: $3 \mathrm{~min}$ at $95^{\circ} \mathrm{C}$, followed by $10 \mathrm{~s}$ at $95^{\circ} \mathrm{C}$ and $30 \mathrm{~s}$ at $58^{\circ} \mathrm{C}$ with 39 cycles, and then was ended after a melt curve analysis from $60^{\circ} \mathrm{C}$ to $95^{\circ} \mathrm{C}$ with an increment of $1{ }^{\circ} \mathrm{C}$ for $4 \mathrm{~s}$ at each step. Three biological replicates were used for each analysis and the data were analyzed using the $2^{-\Delta \Delta \mathrm{Ct}}$ method.

\section{Data statistics}

All the experiments were repeated three times at the biological sample level, and the differences were analyzed using IBM SPSS 19.0 (International Business
Machines Corporation, Armonk, NY, USA) with the Tstudent's method $(P<0.05)$.

\section{Abbreviations}

ACP: Acid phosphatase; AN: Available nitrogen; AP: Available phosphorus; AK: Available potassium; CAT: Catalase; CC: Continuous cropping; CMP: Consecutive monoculture problem; DEGs: Differentially expressed genes; FC: The first cropping; KEGG: The Kyoto Encyclopedia of Genes and Genomes; HPLC: High-performance liquid chromatography; PPO: Polyphenol oxidase; qRT-PCR: quantitative real-time PCR; SC: Sucrase; TK: Total potassium; TP: Total phosphorus; TN: Total nitrogen; UE: Urease

\section{Supplementary Information}

The online version contains supplementary material available at https://doi. org/10.1186/s12870-021-03135-x.

\begin{abstract}
Additional file 1: Fig. S1. Rhizosphere soil phenolic acid detection with HPLC. FC stands for first cropping, and CC stands for continuous cropping. A: standard samples, B: FC soil, C: CC soil. 1: p-hydroxybenzoic, 2: vanillic acid, 3: syringic acid, 4: cumaric acid, 5: ferulic acid.
\end{abstract}

Additional file 2: Table S1. Data output quality summary. Additional file 3: Table S2. Up- and down-regulated DEGs in CC vs FC root tissues.

Additional file 4: Fig. S2. Enriched pathways relative to phenolic acid synthesis.

Additional file 5: Table S3. Primers designed for $\mathrm{QRT} T \mathrm{PCR}$.

Additional file 6: Data of up-regulated DEGs expression level and qRT$P C R$ verification.

Additional file 7: Data of soil enzyme activities in FC and CC rhizosphere soil.

Additional file 8: Data of phenolic acid contents in FC and CC rhizosphere soil. Typical curve of standard samples. Typical curve of CC soil. Typical curve of FC soil.

Additional file 9: Data of heatmap of DEGs relative to phonelic acid metabolism in cc vs fc root.

Additional file 10: Data of chemical properties in FC and CC rhizosphere soil.

\section{Acknowledgements}

We should like to thank Professor Yiwei Mo of College of Life Science, Shaoxing University for reading this manuscript.

\section{Authors' contributions}

NXZ performed most of the experiments. JCZ managed the fields and provided the materials. LAY participated in the preparation of the manuscript. CY and $\mathrm{HYH}$ conceived and coordinated the studies, and $\mathrm{HYH}$ wrote and edited the manuscript. All the authors have read and approved the final manuscript.

\section{Authors' information}

NXZ, JCZ, CY, and HYH work and study in Collaborative Innovation Center for Field Weeds Control of Hunan Province, Hunan University of Humanities, Science and Technology, Loudi 417000, China. LAY works and studies in College of Agronomy, Hunan Agricultural University, Changsha 410128, China.

\section{Funding}

This work was supported by the Scientific Research Project of Public Welfare Industry (Agriculture) (201503232-05) and the Scientific Research Fund of Hunan Provincial Education Department (20A277). The supporters had no role in study design, data collection, data analysis, data interpretation, the writing of the manuscript or decision to publish. 


\section{Availability of data and materials}

All data supporting the findings were contained in the manuscript and its supplementary files except the RNA-seq raw data. And all the RNA-seq raw data were uploaded in the SRA of NCBI (PRJNA507291).

\section{Declarations}

Ethics approval and consent to participate

Not applicable.

\section{Consent for publication}

Not applicable.

\section{Competing interests}

The authors declare that they have no competing interests.

\section{Author details}

'Collaborative Innovation Center for Field Weeds Control of Hunan Province, Hunan University of Humanities, Science and Technology, Loudi 417000, China. ${ }^{2}$ College of Agronomy, Hunan Agricultural University, Changsha 410128, China.

Received: 23 March 2021 Accepted: 25 July 2021

Published online: 07 August 2021

\section{References}

1. Chen Y, Yin L, Zhang X, Wang Y, Chen Q, Jin C, et al. Optimization of alkaline extraction and bioactivities of polysaccharides from rhizome of Polygonatum odoratum. Biomed Res Int. 2014;2014:504896-8. https://doi. org/10.1155/2014/504896.

2. Lan G, Chen H, Wang Z, Zhang W, Zhang L. Extraction of Polygonatum odoratum polysaccharides using response surface methodology and preparation of a compound beverage. Carbohydr Polym. 2011;86(3):117580. https://doi.org/10.1016/j.carbpol.2011.06.009.

3. Li M, Yang Y, Feng F, Zhang B, Yang C, Gu L, et al. Differential proteomic analysis of replanted Rehmannia glutinosa roots by ITRAQ reveals molecular mechanisms for formation of replant disease. BMC Plant Biol. 2017:17(1):116. https://doi.org/10.1186/s12870-017-1060-0

4. Li Z, Wang Q, Ruan X, Pan C, Jiang D. Phenolics and plant allelopathy. Molecules. 2010;15(12):8933-52. https://doi.org/10.3390/molecules15128933.

5. Marchiosi R, dos Santos WD, Constantin RP, de Lima RB, Soares AR, FingerTeixeira A, et al. Biosynthesis and metabolic actions of simple phenolic acids in plants. Phytochem Rev. 2020;11(4):865-906. https://doi.org/10.1007/s111 01-020-09689-2

6. Cheynier V, Comte G, Davies KM, Lattanzio V, Martens S. Plant phenolics: recent advances on their biosynthesis, genetics, and ecophysiology. Plant Physiol Biochem. 2013;72:1-20. https://doi.org/10.1016/j.plaphy.2013.05.009.

7. Weiß S, Winkelmann T. Transcriptome profiling in leaves representing aboveground parts of apple replant disease affected Malus domestica 'M26' plants. Sci Hortic. 2017;222:111-25. https://doi.org/10.1016/j.scienta.2017.05. 012.

8. Xiao L, Liu Y, Xu B, Y Y Y, Liu T. Study on allelopathy and identification of allelochemical in rhizosphere soil of Polygonatum. Chin Agric Sci Bulletin 2015;31:163-7. https://doi.org/10.11924/j.issn.1000-6850.casb15070050.

9. Chen $Y$, Zhang $X$, Hu Y, Jin C. Effects of soil nutrients, enzyme activities and microbial composition in vegetable-Polygonatum Odoratum rotation regimes. Rev Chim. 2020;71(6):460-72. https://doi.org/10.37358/RC.20.6.8212.

10. Zou T, Jin C, Chen Y, Liu Z, Hu Y. Responses of Polygonatum odoratum seedlings in aeroponic culture to treatments of different ammonium: nitrate ratios. J Plant Nutr. 2017;40(20):2850-61. https://doi.org/10.1080/01904167.2 017.1384011.

11. Chen Y, Liu Z, Tu N, Hu Y, Jin C, Luo Y, et al. Integrated transcriptome and microRNA profiles analysis reveals molecular mechanisms underlying the consecutive monoculture problem of Polygonatum odoratum. Cell Mol Biol. 2020;66(2):47-52. https://doi.org/10.14715/cmb/2020.66.2.7.

12. Yang Y, Li M, Li X, Chen X, Lin W, Zhang Z. Transcriptome-wide identification of the genes responding to replanting disease in Rehmannia glutinosa L. roots. Mol Biol Rep. 2015;42(5):881-92. https://doi.org/10.1007/ s11033-014-3825-y.

13. Zhao Y, Cheng Y, Ma Y, Chen C, Xu F, Dong X. Role of phenolic acids from the rhizosphere soils of Panax notoginseng as a double-edge sword in the occurrence of root-rot disease. Molecules. 2018;23(4):819. https://doi.org/1 0.3390/molecules23040819.

14. Li P, Chen J, Li Y, Zhang K, Wang H. Possible mechanisms of control of Fusarium wilt of cut chrysanthemum by Phanerochaete chrysosporium in continuous cropping fields: a case study. Sci Rep. 2017;7(1):15994. https:// doi.org/10.1038/s41598-017-16125-7.

15. Lin $P$, Zhao $H$, Tang Z, Zhang $Y$, Lin $H$, Shen $Y$, et al. Effects of continuous cropping on root exudates of different resistance peanut (Arachis hypogaea L.) varieties and allelochemicals content in soil. Chin J Oil Crop Sci. 2015;37: 467-74. https://doi.org/10.7505/j.issn.1007-9084.2015.04.006.

16. Chen ZL, Krishnamurti GSR, Naidu R. Separation of phenolic acids in soil and plant tissue extracts by co-electroosmotic capillary electrophoresis with direct UV detection. Chromatographia. 2001;53(3-4):179-84. https://doi.org/1 $0.1007 / B F 02491567$.

17. Tian G, Bi Y, Sun Z, Zhang L. Phenolic acids in the plow layer soil of strawberry fields and their effects on the occurrence of strawberry anthracnose. Eur J Plant Pathol. 2015;143(3):581-94. https://doi.org/10.1007/ s10658-015-0711-y.

18. Cecchi AM, Koskinen WC, Cheng HH, Haider K. Sorption-desorption of phenolic acids as affected by soil properties. Biol Fertil Soils. 2004;39(4):23542. https://doi.org/10.1007/s00374-003-0710-6.

19. Lambers H, Chapin FS, Pons TL. Plant physiological ecology. New York: Springer; 1998. https://doi.org/10.1007/978-1-4757-2855-2.

20. Li W, Yang G, Chen H, Tian J, Zhang Y, Zhu Q, et al. Soil available nitrogen, dissolved organic carbon and microbial biomass content along altitudinal gradient of the eastern slope of Gongga Mountain. Acta Ecol Sin. 2013; 33(5):266-71. https://doi.org/10.1016/j.chnaes.2013.07.006.

21. Muzika R. Terpenes and phenolics in response to nitrogen fertilization: a test of the carbon/nutrient balance hypothesis. Chemoecology. 1993;4(1):3-7. https://doi.org/10.1007/BF01245890.

22. Alkorta I, Aizpurua A, Riga P, Albizu I, Amézaga I, Garbisu C. Soil enzyme activities as biological indicators of soil health. Rev Environ Health. 2003; 18(1):65-73. https://doi.org/10.1515/reveh.2003.18.1.65.

23. Zamboni N, Fischer E, Laudert D, Aymerich S, Hohmann H, Sauer U. The Bacillus subtilis yajl gene encodes the NADP+-dependent 6-pgluconate dehydrogenase in the pentose phosphate pathway. J Bacteriol. 2004;186(14):4528-34. https://doi.org/10.1128/JB.186.14.4528-4 534.2004

24. Pickl A, Schönheit $P$. The oxidative pentose phosphate pathway in the haloarchaeon Haloferax volcanii involves a novel type of glucose-6phosphate dehydrogenase - the archaeal Zwischenferment. FEBS Lett. 2015; 589(10):1105-11. https://doi.org/10.1016/j.febslet.2015.03.026.

25. Teige $M$, Melzer M, Süss K. Purification, properties and in situ localization of the amphibolic enzymes D-ribulose 5-phosphate 3-epimerase and transketolase from spinach chloroplasts. Eur J Biochem. 1998;252(2):237-44. https://doi.org/10.1046/j.1432-1327.1998.2520237.x.

26. Ye R, Wong $\mathrm{S}$. Transcriptional regulation of the Bacillus subtilis glucitol dehydrogenase gene. J Bacteriol. 1994;176(3):3314-20. https://doi.org/10.1 007/BF02181950.

27. Roy D, Ward P. Comparison of fructose-1, 6-bisphosphatase gene (fbp) sequences for the identification of lactobacillus rhamnosus. Curr Microbiol. 2004;49(5):313-20. https://doi.org/10.1007/s00284-004-4355-y.

28. Bauer S, Vasu P, Persson S, Mort AJ, Somerville C. Development and application of a suite of polysaccharide-degrading enzymes for analyzing plant cell walls. PNAS. 2006;103(30):11417-22. http://www.pnas.org/cgi/ doi/10.1073/pnas.0604632103.

29. Oliveira JS, Pinto CA, Basso LA, Santos DS. Cloning and overexpression in soluble form of functional shikimate kinase and 5-enolpyruvylshikimate 3phosphate synthase enzymes from mycobacterium tuberculosis. Protein Expr Purif. 2001;22(3):430-5. https://doi.org/10.1006/prep.2001.1457.

30. Charles IG, Lamb HK, Pickard D, Dougan G, Harwkins AR. Isolation, characterization and nucleotide sequences of the aroC genes encoding chorismate synthase from Salmonella typhi and Escherichia coli. J Gen Microbiol. 1990;136(2):353-8. https://doi.org/10.1099/00221287-136-2-353.

31. Leung PSC, Preiss J. Biosynthesis of bacterial glycogen: primary structure of Salmonella typhimurium ADPglucose synthetase as deduced from the nucleotide sequence of the glgC gene. J Bacteriol. 1987;169(1):4355-60. https://doi.org/10.1083/jcb.107.1.101.

32. Yoshida K, Komae K. A rice family 9 glycoside hydrolase isozyme with broad substrate specificity for hemicelluloses in yype II cell walls. Plant Cell Physiol. 2006;47(11):1541-54. https://doi.org/10.1093/pcp/pcl020. 
33. Froman BE, Tait RC, Gottlieb LD. Isolation and characterization of the phosphoglucose isomerase gene from Escherichia coli. Mol Gen Genet. 1989;217(1):126-31. https://doi.org/10.1007/BF00330951.

34. Mandal ST, Chakraborty D, Dey S. Phenolic acids act as signaling molecules in plant-microbe symbioses. Plant Signal Behav. 2010;5(4):359-68. https:// doi.org/10.4161/psb.5.4.10871.

35. Becerra-Moreno A, Redondo-Gil M, Benavides J, Nair V, Cisneros-Zevallos L, Jacobo-Velázquez. Combined effect of water loss and wounding stress on gene activation of metabolic pathways associated with phenolic biosynthesis in carrot. Front Plant Sci. 2015;6:837. https://doi.org/10.3389/ fpls.2015.00837.

36. Li Y, Wang C, Wang F, Dong H, Guo S, Yang JX. Phenolic components and flavanones from Dendrobium andidum. Chin Pharm J. 2010;45:975-9.

37. Cho M, Lee S. Phenolic phytoalexins in rice: biological functions and biosynthesis. Int J Mol Sci. 2015;16(12):29120-33. https://doi.org/10.3390/ ijms161226152.

38. Park LH, Lee S, Jung K, Hahn T, Cho M. Transcriptomic analysis of UV-treated rice leaves reveals UV-induced phytoalexin biosynthetic pathways and their regulatory networks in rice. Phytochem. 2013;96:57-71. https://doi.org/10.1 016/j.phytochem.2013.08.012.

39. Riley D, Barber SA. Bicarbonate accumulation and $\mathrm{pH}$ changes at the soybean (Glycine max (L.) Merr.) root-soil interface. Soil Sci Soc Am J. 1969: 33(6):905-8. https://doi.org/10.2136/sssaj1969.03615995003300060031x.

40. Riley D, Barber SA. Salt accumulation at the soybean (Glycine max ( L.) Merr.) root-soil interface. Soil Sci Soc Am J. 1970;34(1):154-5. https://doi.org/10.213 6/sssaj1970.03615995003400010042x

41. Wang J, Zhang L, Guo Q, Kou J, Yu B, Gu D. Quantitative analysis of seven phenolic acids in eight Yinqiao Jiedu serial preparations by quantitative analysis of multi-components with single-marker. Acta Pharm Sin. 2015; 50(4):480-5. https://doi.org/10.16438/j.0513-4870.2015.04.001.

42. Zheng B. Technical guide for soil analysis. Beijing: China Agricultural Press; 2012. p. 154-212.

43. Arogba SS, Ajiboye OL, Ugboko LA, Essienette SY, Afolabi PO. Properties of polyphenol oxidase in mango (Mangifera indica) kernel. J Sci Food Agric 1998;77(4):459-62. https://doi.org/10.1002/(SICI)1097-0010(199808)77:4< 459::AID-JSFA61>3.0.CO;2-O.

44. Kraus TE, Fletcher RA. Paclobutrazol protect wheat seedlings from heat and paraquat injury. Is detoxification of active oxygen involved? Plant Cell Physiol. 1994;35(1):45-52. https://doi.org/10.1159/000163877.

45. Das S, Ganguly D, Mukherjee A, Chakraborty S, De TK. Soil urease activity of Sundarban mangrove ecosystem, India. Adv Microbi. 2017;7(8):617-32. https://doi.org/10.4236/aim.2017.78048.

46. Saa A, Trasar-Cepeda MC, Gil-Sotres F, Carballas T. Changes in soil phosphorus and acid phosphatase activity immediately following forest fires. Soil Biol Biochem. 1993;25(9):1223-30. https://doi.org/10.1016/0038-071 7(93)90218-Z.

47. Li Z, Lu Z. Soil Sucrase: detection conditions based on DNS colorimetric. Chin Agric Sci Bulletin. 2016;32:171-6.

48. Grabherr MG, Haas BJ, Yassour M, Levin JZ, Thompson DA, Amit I, et al. Fulllength transcriptome assembly from RNA-Seq data without a reference genome. Nat Biotechnol. 2011;29(7):644-52. https://doi.org/10.1038/nbt.1 883.

49. Anders S, Huber W. Differential expression analysis for sequence count data. Genome Biol. 2010;11(10):R106. https://doi.org/10.1186/gb-2010-11-10-r106.

50. Mao X, Cai T, Olyarchuk JG, Wei L. Automated genome annotation and pathway identification using the KEGG Orthology (KO) as a controlled vocabulary. Bioinf. 2005;21(2):3787-93. https://doi.org/10.2307/1592215.

\section{Publisher's Note}

Springer Nature remains neutral with regard to jurisdictional claims in published maps and institutional affiliations.

\section{Ready to submit your research? Choose BMC and benefit from}

- fast, convenient online submission

- thorough peer review by experienced researchers in your field

- rapid publication on acceptance

- support for research data, including large and complex data types

- gold Open Access which fosters wider collaboration and increased citations

- maximum visibility for your research: over $100 \mathrm{M}$ website views per year

At BMC, research is always in progress.

Learn more biomedcentral.com/submissions 